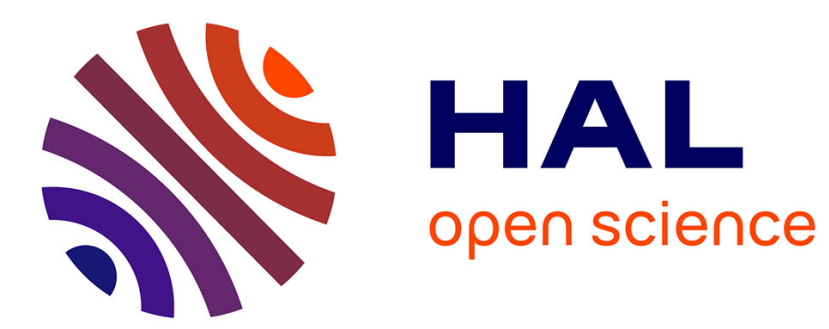

\title{
La relocalisation des approvisionnements de la restauration collective et le code des marchés publics \\ Ronan Le Velly
}

\section{To cite this version:}

Ronan Le Velly. La relocalisation des approvisionnements de la restauration collective et le code des marchés publics. Pour : enquêtes et témoignages, 2012, 215-216, pp.269-274. hal-00773568

\section{HAL Id: hal-00773568 \\ https://hal.science/hal-00773568}

Submitted on 14 Jan 2013

HAL is a multi-disciplinary open access archive for the deposit and dissemination of scientific research documents, whether they are published or not. The documents may come from teaching and research institutions in France or abroad, or from public or private research centers.
L'archive ouverte pluridisciplinaire HAL, est destinée au dépôt et à la diffusion de documents scientifiques de niveau recherche, publiés ou non, émanant des établissements d'enseignement et de recherche français ou étrangers, des laboratoires publics ou privés. 
Pour, n 215-216, décembre 2012, p. 269-274.

Note : la version publiée dans Pour comporte une erreur. Page 273, il convient de lire 15000 euros et non pas 4000 euros.

\section{La relocalisation des approvisionnements de la restauration collective et le code des marchés publics}

\section{Ronan Le Velly, Maître de conférences en sociologie, Montpellier SupAgro, UMR Innovation}

Le code des marchés publics est-il compatible avec la relocalisation des approvisionnements de la restauration collective ? Alors qu'il y a cinq ans à peine, une telle question ne suscitait guère d'intérêt, elle est aujourd'hui devenue pleinement d'actualité. Elle est traitée dans de nombreux guides pratiques publiés par des collectivités territoriales, des organisations du monde agricole, des experts et des chercheurs... Des formations sont organisées dans les régions, à destination aussi bien des agriculteurs ou agents de développement que des gestionnaires ou cuisiniers de la restauration collective. Des responsables politiques locaux et nationaux se positionnent sur le sujet, pour appeler à un changement de pratiques ou à une évolution du droit, etc. Ainsi, en quelques années, une masse d'argumentaires, d'expériences et d'expertises a été produite sur la place des achats alimentaires locaux dans les marchés publics.

Dans ce court article, je n'ambitionne pas de faire la synthèse de ces connaissances. En outre, je ne suis pas juriste mais sociologue et mes recherches portent moins sur le contenu du droit que sur les démarches engagées sur le terrain. Dans cette perspective, la thèse que je défendrai est que la relocalisation des achats de la restauration collective publique n'est pas qu'une question d'ordre juridique. Si les interdits et les possibles du code des marchés se doivent d'être compris (section 1), la rédaction d'un appel d'offres par une collectivité désireuse de travailler avec des producteurs locaux nécessite aussi une connaissance mutuelle entre ces deux groupes (section 2). En outre, les achats publics de produits locaux se font aussi en dehors des appels d'offres, avec des effets qu'il convient d'observer avec attention (section 3 ).

\section{L'interdiction d'exiger ou de valoriser la proximité géographique en tant que telle}


Le code des marchés publics a connu d'importantes évolutions ces quinze dernières années. Tout d'abord, depuis 2001, les marchés ne sont plus automatiquement attribués au candidat le moins cher. Dans un souci d'accroître l'efficacité des achats, la procédure vise aujourd'hui à sélectionner «l'offre économiquement la plus avantageuse », en tenant compte du prix et d'autres critères déterminés selon l'objet du marché. En 2004 et 2006, deux nouvelles réformes du code des marchés publics ont également rendu possible d'inclure des critères de développement durable. Cette possibilité a été le fruit d'une longue évolution de la jurisprudence nationale et communautaire : tenir compte des performances sociales et environnementales des fournisseurs a longtemps été considéré comme une pratique discriminatoire, contraire à la liberté d'accès et l'égalité de traitement (Schiesser et Cantillon, 2007). Ce n'est aujourd'hui plus le cas. Des objectifs de développement durable peuvent être inclus dans la définition du besoin (art. 5), les spécifications techniques (art. 6), les conditions d'exécution du marché (art. 14) et les modalités de sélection de «l'offre économiquement la plus avantageuse $»($ art. 53).

Ce cadre permet d'exiger des produits issus de l'agriculture biologique ou d'un mode de production «équivalent» comme Demeter ou Nature et Progrès. Il permet aussi de choisir entre différents fournisseurs de denrées alimentaires en tenant compte de leurs performances sociales et environnementales: leurs offres sont alors évaluées à partir d'une grille multicritères incluant par exemple la politique de recyclage des emballages ou le recours à des travailleurs en insertion. Dans un marché ouvert à des produits bio et non bio, il est de la même façon possible de considérer le prix à hauteur de $\mathrm{x} \%$ de la note finale et l'écologie du mode de production à hauteur de y \%. Selon l'importance de ces pondérations, une offre de produits bio, même plus onéreuse, peut alors remporter le marché.

Le droit a donc beaucoup évolué, mais il ne permet pas pour autant d'exiger une origine locale pour les produits ou services achetés. Sur ce point, les principes de liberté d'accès et d'égalité de traitement prévalent toujours. Néanmoins, la proximité géographique peut parfois être considérée comme un élément de preuve quant à la capacité du candidat à correctement répondre aux demandes exprimées dans l'appel d'offres. En avril 2007, dans une affaire portant sur un marché de transport public de personnes, il a ainsi été jugé par la Cours administrative d'appel de Marseille que le lieu de stationnement du parc de matériel des candidats peut constituer un élément objectif d'appréciation de la qualité des prestations. Pour les denrées alimentaires, il est de même possible d'envisager la proximité géographique comme un élément venant attester de meilleures performances en matière de fraîcheur des 
produits, de réactivité de livraison, de réduction des émissions de gaz à effet de serre ou de respect de la saisonnalité. Mais, de nouveau, elle ne peut être exigée en tant que telle. Rien ne garantit alors qu'un fournisseur non-local, ayant de meilleures performances sur ces points, remporte le marché. C'est précisément ce qui atteste du caractère non discriminatoire de la procédure.

En 2011, le Ministre de l'agriculture Bruno Le Maire avait à plusieurs reprises annoncé son intention de changer le code des marchés publics pour inclure la «proximité de la production » dans les critères de choix reconnus par l'article 53. Le décret du Ministre de l'Economie du 26 août 2011 a effectivement modifié cet article, mais en s'en tenant à la possibilité de prendre en compte «les performances en matière de développement des approvisionnements directs de produits de l'agriculture ». En permettant de valoriser la réduction du nombre d'intermédiaires au moment de la sélection des offres, ce nouveau critère permet d'envisager de cibler des producteurs locaux. Mais, de nouveau, ce n'est pas la proximité géographique en tant que telle qui est valorisée. L'évolution du code est donc moins ambitieuse qu'annoncée, mais aussi moins juridiquement incertaine, tant un critère de préférence locale risquait d'être jugé contraire aux règles communautaires de liberté de la concurrence.

\section{Un besoin de connaissance mutuelle entre les acteurs de l'offre et de la demande}

Le code des marchés publics constitue donc une réelle entrave à l'achat public local. Mais, la section précédente le laissait déjà entrevoir, il offre aussi certaines opportunités. En particulier, choisir des critères adéquats d'évaluation des offres peut permettre de valoriser les performances des producteurs locaux en termes de réactivité, de fraîcheur, de respect de la saisonnalité, d'émissions de gaz à effet de serre ou de relation directe ; tout du moins lorsque leurs performances sont effectivement supérieures à celles des producteurs non-locaux. Cette piste mérite d'être empruntée. Elle est d'ailleurs mise en avant dans nombre de guides pratiques (Corabio, 2010 ; DRAAF Rhône-Alpes, 2010 ; Ducoeurjoly, 2010 ; FN CIVAM, Fondation Nicolas Hulot, 2009 ; Réseau Grand Ouest, 2010). Néanmoins, elle ne doit pas amener à poser uniquement le problème en termes de contraintes et d'opportunités juridiques. Pour moi, ce constat a été particulièrement manifeste à l'observation en 2009 d'un groupe de travail de collectivités territoriales de l'ouest de la France. A cette époque, peu de documentation était disponible sur le sujet de l'achat public bio et local. Un juriste et un acheteur, de la ville d'Angers et de Niort, avaient alors été missionnés par le groupe de travail 
pour étudier les possibles en la matière. Lors de leurs premières restitutions, ils ont répondu à cette question dans des termes strictement juridiques. Mais, suite à des rencontres avec des organisations de producteurs locaux, leur discours a beaucoup évolué. Dans leur restitution finale, il apparaissait clairement qu'une bonne compréhension du droit devait se coupler avec une bonne connaissance des caractéristiques de l'offre disponible sur le territoire.

Pour prendre un exemple simple, il est régulièrement avancé que l'allotissement est une modalité d'appel d'offres pertinente pour développer un achat de proximité. Il est effectivement rare que des producteurs locaux disposent d'une gamme de produits aussi étendue que les entreprises de distribution qui fournissent habituellement la restauration collective. Néanmoins, pour réaliser cet allotissement de façon pertinente, il est impératif de connaître la nature de l'offre locale (type de produits, conditionnement, prix...) et les contraintes qui lui sont associées (saisonnalité, variabilité...), sous peine que les producteurs régionaux ne répondent même pas à l'appel d'offres ou fassent des propositions hors de prix. De même, rien ne sert d'exiger un délai maximum de trois jours entre la récolte et la livraison, avec l'idée de privilégier les producteurs locaux, si l'on n'est pas certain que ces derniers sont non seulement capables mais aussi désireux de le faire.

Le respect du code des marchés publics n'est pas une question centrale et isolée, mais une question parmi d'autres. Les conditions logistiques, la nature et durée du contrat, le degré de transformation du produit... tous ces paramètres doivent être abordés de concert. Or, dans beaucoup de situations, un état de méconnaissance croisée entre les acteurs de l'offre et les acteurs de la demande complique l'établissement d'un accord sur ces différents points. Les retours d'expérience témoignent alors des bénéfices des rencontres entre producteurs locaux et agents de collectivités. Discuter autour d'une table, visiter les exploitations des uns et les cuisines des autres, permet de mettre un visage derrière un produit ou un client, de lever nombre d'a priori et d'incompréhensions, de mettre à plat les contraintes respectives et d'établir les compromis nécessaires (Le Velly et Bréchet, 2011). La rédaction d'un appel d'offres gagne alors à être considérée comme l'aboutissement d'un cheminement, plutôt que comme le point de départ des échanges.

\section{Tout ne se passe pas dans le cadre des appels d'offres}

En pratique, beaucoup se passe également en dehors des appels d'offres. Tout d'abord, lorsque le montant commandé n'excède pas les 15000 euros, l'achat public peut se faire sans 
cette procédure. Cette situation est très commune pour les petites collectivités, qui achètent au coup par coup auprès d'agriculteurs locaux. Ensuite, certaines collectivités mettent en avant le caractère expérimental d'un achat local pour, ponctuellement, justifier de ne pas commander au fournisseur à qui elles ont attribué le marché concerné. Cette démarche est supposée être temporaire, mais il arrive qu'elle s'inscrive dans la durée sans que le fournisseur ne la conteste pour ne pas fâcher la collectivité avec qui il travaille. Enfin, beaucoup se négocie de façon informelle, à côté de la procédure officielle. Il est par exemple possible pour une collectivité d'exprimer à un distributeur ou un grossiste son souhait d'être livrée en produits régionaux de saison. Au moment du renouvellement du marché, le message peut également être transmis en exigeant des candidats un mémoire technique décrivant leurs schémas d'approvisionnement.

Les solutions précédentes ont l'avantage de la simplicité, mais elles doivent être observées avec un regard critique. La contractualisation induite par la procédure d'appels d'offres a aussi des avantages, surtout lorsqu'elle s'inscrit dans la durée. Les achats ponctuels et les achats expérimentaux offrent ainsi peu de visibilité aux producteurs. Certes, dans de nombreux cas, les volumes sont faibles et les enjeux pour l'exploitation peu importants. Mais dans d'autres, le producteur peut se trouver engagé dans une relation qui compte dans son organisation agronomique et économique. De la même façon, lorsqu'une collectivité obtient d'un fournisseur qu'il se tourne vers des produits locaux, il lui faut veiller à la continuité de cette orientation, quitte à devoir le rappeler à l'ordre lorsqu'il ne respecte pas sa promesse.

Les délégations de service public posent le même type de problèmes. D'un côté, elles offrent un cadre réglementaire favorable. Les achats des sociétés (privées) de restauration collective ne sont pas régis par le code des marchés publics et peuvent se tourner vers des producteurs locaux sans difficultés juridiques. Pour la collectivité, il s'agit alors simplement de vérifier que le fait d'exiger des menus incluant des produits locaux n'induit pas de discrimination entre les sociétés de restauration; ce qui est généralement le cas. D’un autre côté, dans cette organisation la collectivité perd le contrôle des achats alimentaires. Le risque est alors que la société de restauration ne joue pas totalement le jeu, en ne respectant pas son engagement d'achat local ou en usant de pratiques commerciales agressives envers les producteurs. La collectivité se doit donc de suivre au plus près les pratiques de son prestataire, de mettre en place des dispositifs ad hoc de contrôle (embaucher le chef de cuisine...) et/ou de dialogue (comité de suivi multipartites...). 


\section{Conclusion}

Interroger les achats publics de denrées alimentaires locales au regard du code des marchés publics peut être une bonne comme une mauvaise entrée pour la réflexion et pour l'action. Elle est mauvaise si elle conduit à poser le problème dans des termes strictement juridiques, négligeant les dynamiques collectives et les enjeux socio-économiques associés à ces démarches. Mais ce questionnement peut aussi être une entrée concrète et fructueuse. S'il amène à débattre dans les territoires de l'organisation de la production, des échanges et de l'alimentation, son potentiel transformateur peut même être non-négligeable.

\section{Références}

Corabio, Guide pratique de la restauration collective bio en Rhône-Alpes, 2010.

DRAAF Rhône-Alpes, Favoriser une restauration collective de proximité et de qualité. 2010.

Delphine Ducoeurjoly, Guide pratique pour une restauration collective bio. Territorial éditions, 2010.

Fédération Nationale des CIVAM, Fondation Nicolas Hulot pour la Nature et l'Homme, Guide de la restauration collective responsable, à l'attention des collectivités et des entreprises, 2009.

Ronan Le Velly, Jean-Pierre Bréchet, Le marché comme rencontre d'activités de régulation. Initiatives et innovations dans l'approvisionnement bio et local de la restauration collective. Sociologie du travail 53 (4), pp. 478-492, 2011.

Réseau Grand Ouest, Intégration de produits issus de l'agriculture biologique dans la restauration collective, 2010.

Philippe Schiesser, Guillaume Cantillon, L'achat public durable. Outils et méthode pour réussir des achats publics respectueux du développement durable. Le Moniteur, 2007. 